\title{
Preface to special issue: LINEARITY 2014
}

\author{
SANDRA ALVES $†$ and ILIANO CERVESATO \\ $\dagger$ CRACS/INESCTEC \& Faculty of Science, University of Porto, Portugal \\ Email: sandra@dcc.fc.up.pt \\ $\ddagger$ Computer Science Department, Carnegie Mellon University, Pittsburgh, Pennsylvania, U.S.A. \\ Email: iliano@cmu.edu
}

Received 26 October 2016

This special issue collects selected articles from the Third International Workshop on Linearity (LINEARITY 2014), which was held in Vienna, on July 13th, 2014. The workshop was a one-day satellite event of FLoC 2014, the sixth Federated Logic Conference, which was held as part of the 2014 Vienna Summer of Logic.

The aim of the International Workshop on Linearity series is to provide a forum where researchers exploring theory and applications of linear calculi can present ideas and work in progress, as a mean to foster interaction, whilst at the same time enabling newcomers to learn about active areas of research. In a world searching for robust, verifiable and efficient programming languages, the ability to have more control on computational resources has become increasingly important. Steeming from Jean-Yves Girard seminal work on linear logic, linearity plays a key role in a variety of areas of Computer Science and influences our work on proof technology, complexity classes, quantum computation, program analysis, expressive operational semantics, linear programming languages, techniques for program transformation, update analysis and efficient implementation, just to cite a few. The workshop has welcomed new results that made a central use of linearity, ranging from foundational work to applications in any field. This included sub-linear logics, linear term calculi, linear type systems, linear proof-theory, linear programming languages, applications to concurrency, interaction-based systems, verification of linear systems, quantum models of computation and biological and chemical models of computation, amongst others.

This issue contains four articles, out of the 10 papers selected for presentation at LINEARITY 2014, representing different aspects of linearity. The first article, by Taus Brock-Nannestad and Nicolas Guenot, entitled 'Multifocused Cut Elimination,' presents a multifocussed sequent calculus for polarized linear logic, and proves the system satisfies cut elimination, taking advantage of the design choices of the calculi. The authors further discuss the effect of cut elimination on the structure of proofs, which exploits the strong structure of multifocussed proofs. The next article, 'Expressing Additives Using Multiplicatives and Subexponentials,' by Kaustuv Chaudhuri, shows that the extension of classical propositional multiplicative linear logic with subexponentials is undecidable by providing an encoding of the halting problem for two-register Minsky machines. It also shows that the combination of multiplicatives and subexponentials in this logic permits defining the additive operators of linear logic as derived connectives. The article by Marco Solieri, entitled 'Geometry of Resource Interaction and Taylor-Ehrhard-Regnier Expansion - A Minimalist Approach,' studies the interplay between the dynamics of beta-reduction 
through the Geometry of Interaction (GOI), and the Taylor expansion of beta-reduction by means of the Resource $\lambda$-calculus (RC). This is achieved by defining an appropriate notion of persistent paths in a strictly typed version of RC and a suitable GOI for $\mathrm{RC}$, that is invariant under reduction, and that counts addends in normal forms. The article further analyses expansion on paths in ordinary terms, showing that persistence can be transferred back and forth between a path and its expansion. Finally, the article by Jennifer Paykin and Steve Zdancewic on 'A Linear/Producer/Consumer model of Classical Linear Logic,' presents a linear/producer/consumer (LPC) logic that puts the three classes of propositions on the same semantic footing, following Benton's linear/non-linear formulation of intuitionistic linear logic. Semantically, LPC corresponds to a system of three categories connected by adjunctions reflecting the linear/producer/consumer structure. The results in the article include admissibility theorems for the cut and duality rules, a translation of the LPC logic into category theory, as well as several concrete instances of the LPC model.

We would like to thank all involved in this special issue. First, we would like to thank all authors for submitting their contributions and for the care and attention they took in subsequent revisions. We thank all the anonymous referees for their thorough and careful reviews. We thank all the members of MSCS involved in processing this issue. Last but not least we would like to express our gratitude to the former and current MSCS editors, Giuseppe Longo and Pierre-Louis Curien, for their support in making this issue possible.

Guest editors of the special issue:

Sandra Alves

Iliano Cervesato 